\title{
Respuesta ambiental en el Pacífico frente a la subducción de la dorsal asísmica de Cocos (Panamá y Costa Rica)
}

\section{Carlos De Gracia ${ }^{1 *}$, Aaron O’Dea ${ }^{1}$, Félix Rodríguez ${ }^{1} \&$ Luis D’ $\mathrm{Croz}^{2}$}

1. Smithsonian Tropical Research Institute, Apartado postal 0843 casilla 03092 Panamá, República de Panamá; degraciac@gmail.com, aaronodea@gmail.com,rodriguezf@si.edu

2. Depto. Biología Marina y Limnología, Facultad de Ciencias Naturales y Exactas, Universidad de Panamá, El Cangrejo, Ciudad de Panamá, Panamá; dcrozlc@gmail.com

\author{
Recibido 11-VII-2011. Corregido 14-XI-2011. Aceptado 14-XII-2011.
}

\begin{abstract}
Environmental response in the Pacific to aseismic Cocos Ridge subduction (Panama and Costa Rica). The evolution of the marine communities along the Pacific coast of Central America, may have changed in response to the formation of the Isthmus of Panama. To evaluate the effect of the Aseismic Cocos Ridge (DAC) subduction on the marine benthic communities, we reconstructed benthic assemblages from Neogene fossiliferous formations in Burica and Nicoya peninsulas of Panama and Costa Rica. Paleoecological and paleoenvironmental conditions were reconstructed by comparing community structure from bulk fossil samples with dredge collections from modern Tropical American seas, using principal component analysis. Our results indicate that during the early Pliocene, before the closing of the Isthmus, some oceanic islands existed with moderate upwelling in the Burica region. After the closure, during the late Pliocene and early Pleistocene the collision of the DAC caused an uplift of the seafloor, where water depth of $2300 \mathrm{~m}$ became shallow waters of less than 40m depth. Meanwhile, upwelling intensified in the open ocean the uplift that had formed small islands in coastal areas of Burica, creating protected areas and limiting the upwelling effect that was given in open ocean. The subduction of the DAC continued until the islands were joined to the mainland and gradually disappeared, allowing the return of the upwelling. During the middle Pleistocene a second process of accelerated uplift with speeds of $8 \mathrm{~m} / 1000$ years provoked again the elevation of the seafloor and later the elevation of the Talamanca Range. The new range formed a barrier that blocked the passage of the Trade winds, created new ecological conditions and optimized and allowed the growth of the best coral reefs in the coasts of the tropical Eastern Pacific (POT) between Panama and Costa Rica. Rev. Biol. Trop. 60 (2): 893-908. Epub 2012 June 01.
\end{abstract}

Key words: Talamanca Range, closure of Panama isthmus, upwelling, benthic communities, Pacific Ocean, marine fossils.

Cuando el Istmo de Panamá se cerró hace 3.5Ma, el Océano Pacífico Oriental (POT) quedó separado del Mar Caribe (Coates et al. 1992, 2004, Coates 1997, Coates \& Obando 1996) y ocurrieron drásticos cambios ambientales producto del colapso del afloramiento (O’Dea et al. 2007a). Este evento condujo a extinciones progresivas de la fauna bentónica por el colapso en la productividad del plancton (Jackson et al. 1993, Jackson 1994, Collins et al. 1996, Coates \& Obando 1996, Jackson et al. 1996, Jackson \& D'Croz 1997, Kameo 2002, Todd et al. 2002, Jain \& Collins 2007,
O'Dea et al. 2007, O'Dea \& Jackson 2009, Smith \& Jackson 2009). La productividad en este océano era causada por la alta concentración de nutrientes transportados por las masas de agua que fluían en dirección este a través del canal interoceánico (O'Dea et al. 2007a). Las aguas que fertilizaban el Mar Caribe mantenían de esta manera, comunidades bentónicas compuestas principalmente por moluscos filtradores que prosperaban por la alta disponibilidad de nutrientes (Jackson et al. 1993, Jackson \& D'Croz 1997, Todd et al. 2002, O'Dea et al. 2007a). 
Aunque el afloramiento cesó en el Caribe después del cierre del Istmo, hoy sigue ocurriendo en el Pacífico Oriental Tropical (POT), específicamente en los golfos de Tehuantepec, Papagayo, Nicoya y Panamá (Fig. 1) (Jackson \& D'Croz 1997, Brenes et al. 2003). Sin embargo, en el resto de las costas del POT esto no ocurre porque los vientos Alisios (provenientes del noreste) responsables de las surgencias, son bloqueados por las altas cadenas montañosas de Centroamérica (Jackson \& D’Croz 1997). Por ejemplo, entre Panamá y Costa Rica, las zonas costeras que se extienden desde el Valle de Antón hasta el Arenal por la costa pacífica no presentan evidencias de surgencias marino-costeras porque la cordillera de Talamanca bloquea los vientos Alisios (Glynn 1977, Coates \& Obando 1996, Coates
1997, Jackson \& D'Croz 1997, D’Croz \& O’Dea 2007, D'Croz \& O'Dea 2009). A causa de esto se desarrollan en estos lugares los mayores arrecifes costeros del POT ya que las costas están protegidas de los vientos (Glynn et al. 1972, Porter 1972, Guzmán \& Cortés 1994, Glynn et al. 1996, Cortés 1997, Glynn \& Maté 1997, Jackson \& D'Croz 1997, Cortés \& Jiménez 2003, Maté 2003). No obstante, la historia geológica indica que aparentemente estas condiciones son de origen muy reciente porque las montañas que bloquearon los vientos no existían antes de la subducción de la DAC, por lo menos desde 5Ma (Alvarado et al. 2007, Collins et al. 1995, Coates 1997).

Nuevas investigaciones indican que la cordillera inició su elevación desde el Pleistoceno temprano, intensificándose durante el

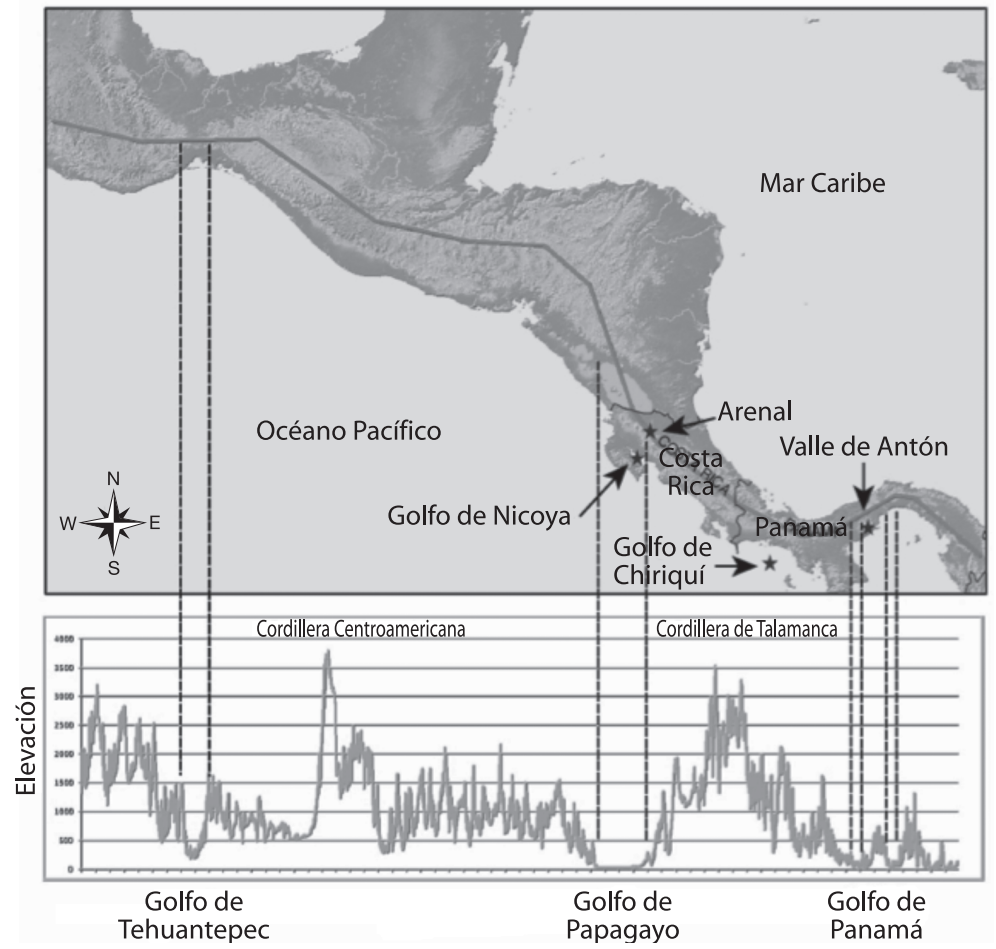

Fig. 1. Imagen construida con sistema de información geográfica donde se muestra la elevación de las montañas en América Central. En los puntos donde la cordillera es baja como el Golfo de Tehuantepec, el Golfo de Papagayo y el Golfo de Panamá, los vientos Alisios pueden pasar y provocan el afloramiento. Pero la cordillera de Talamanca y la cordillera Centroamericana bloquean el paso de los vientos en los puntos donde las montañas son altas.

Fig. 1. Constructed image with geographic information system that shows the elevation of the mountains in Central America. At the points where the mountains are low how the Gulf of Tehuantepec, Gulf of Papagayo and Gulf of Panama the trade winds can pass and cause the upwelling. 
Pleistoceno tardío (León-Rodríguez \& Collins 2005, León-Rodríguez 2007). Por esta razón, creemos que el levantamiento de la cordillera guarda una fuerte influencia sobre las condiciones ambientales que existen hoy día en las zonas sin afloramiento de las costas del POT en Panamá y de Costa Rica. El saber cómo se organizaron las comunidades en el POT en el pasado representa un vacío en la historia natural del cierre del Istmo de Panamá. A pesar de que ya contamos con múltiples estudios para el Caribe, desconocemos qué sucedió en el POT en las etapas finales de este evento. Nuestro trabajo constituye el primer estudio cuantitativo de paleoecología para el POT siguiendo las metodologías estandarizadas por el Proyecto de Paleontología de Panamá (Collins \& Coates 1999, O’Dea et al. 2007b).

\section{MATERIALES Y MÉTODOS}

Descripción geológica: Actualmente la Cordillera Centroamericana y la Cordillera de Talamanca bloquean el paso de los vientos Alisios pero estos pueden pasar en las zonas de baja elevación y provocan el afloramiento en las aguas del POT (Fig. 1). Para entender el efecto que tuvo la elevación de la Cordillera de Talamanca sobre los ambientes del POT estudiamos sitios fosilíferos puntuales en cuatro formaciones geológicas. Tres ubicados en la Península Burica, que corresponden al grupo Charco Azul (Olsson 1942) como son las formaciones: Peñita (>3.5Ma), Burica (2.7Ma) y Armuelles (1.8Ma) (Coates et al. 1992) y una ubicada en la Península Nicoya: la Formación Montezuma (<1.7) (Fig. 2 A-D).

La Formación Peñita es la más antigua del grupo Charco Azul, le infrayacen basaltos volcánicos del Cenozoico y la suprayace la Formación Burica (Fig. 2B, D) (Coates et al. 1992). Esta tiene una espesura compuesta de aproximadamente $1200 \mathrm{~m}$ de sedimentos (Corringan et al. 1990) y se caracteriza por la presencia de limolitas y litoarenitas arcillosas de color azul verdoso que todo el tiempo son del tipo glauconíticas (Coates et al. 1992). La unidad basal posee cuarzo, y litoarenitas de color azul verdoso, con canales locales de conglomerados volcánicos (Fig. 2D). El miembro La Cancha consiste en una serie de facies con estratigrafía cruzadas cuneiformes, litoarenitas con conglomerados y camas cruzadas de areniscas finas con clastos de lodo (Fig. 2B, D). Los afloramientos portadores de fósiles que nosotros estudiamos se encuentran en el Río la Peña ( $8^{\circ} 12.794^{\prime} \mathrm{N}$ - 8256.820’ W), así como en el cauce y en la desembocadura de la quebrada Peñita $\left(8^{\circ} 14.283^{\prime} \mathrm{N}-82^{\circ} 57.852^{\prime}\right.$ W) (Fig. 2B), donde se localiza el lugar de contacto de la Formación Peñita con el basalto volcánico (Fig. 2D). La Peñita ocasionalmente es rica en moluscos siendo los más frecuentes los bivalvos Arca y Chione, y los gasterópodos Olivella, Conus y Cancellaria (Olsson 1942, O'Dea et al. 2007b), también son comunes los cirrípedos de aguas intermareales y numerosas espinas de erizos Eucidaris (O'Dea et al. 2007b, De Gracia 2009). Los paleoambientes indican que los sedimentos se depositaron en aguas poco profundas y que la base de la formación se depositó alrededor de una isla o montaña submarina con en ambiente marino marginal (Coates et al. 1992).

La segunda, es la Formación Burica (Fig. 2 ), que consiste en una sección con alrededor de $2800 \mathrm{~m}$. Las facies son productos de múltiples ciclos con depósitos de turbidita, con megabrechas que están presentes desde la base de la Formación (Corringan et al. 1990, Coates et al. 1992). La Formación Burica está subdividida en dos miembros. El miembro inferior data del Plioceno superior y está compuesto por turbiditas proximales de grano grueso, fue depositado entre 2000 y $2400 \mathrm{~m}$ de profundidad (Coates et al. 1992, Collins et al. 1995). El miembro superior de edad Pleistoceno temprano, está constituido de turbiditas distales de grano fino con conglomerados ricos en fósiles de aguas someras (Fig. 2B, D) (De Gracia 2009, Corringan et al. 1990, Coates et al. 1992). La paleobatimetría de la Formación Burica pasa gradualmente por una transición desde aguas muy profundas hasta aguas poco profundas que caracterizan el miembro superior (Coates et al. 1992). A esta Formación le 


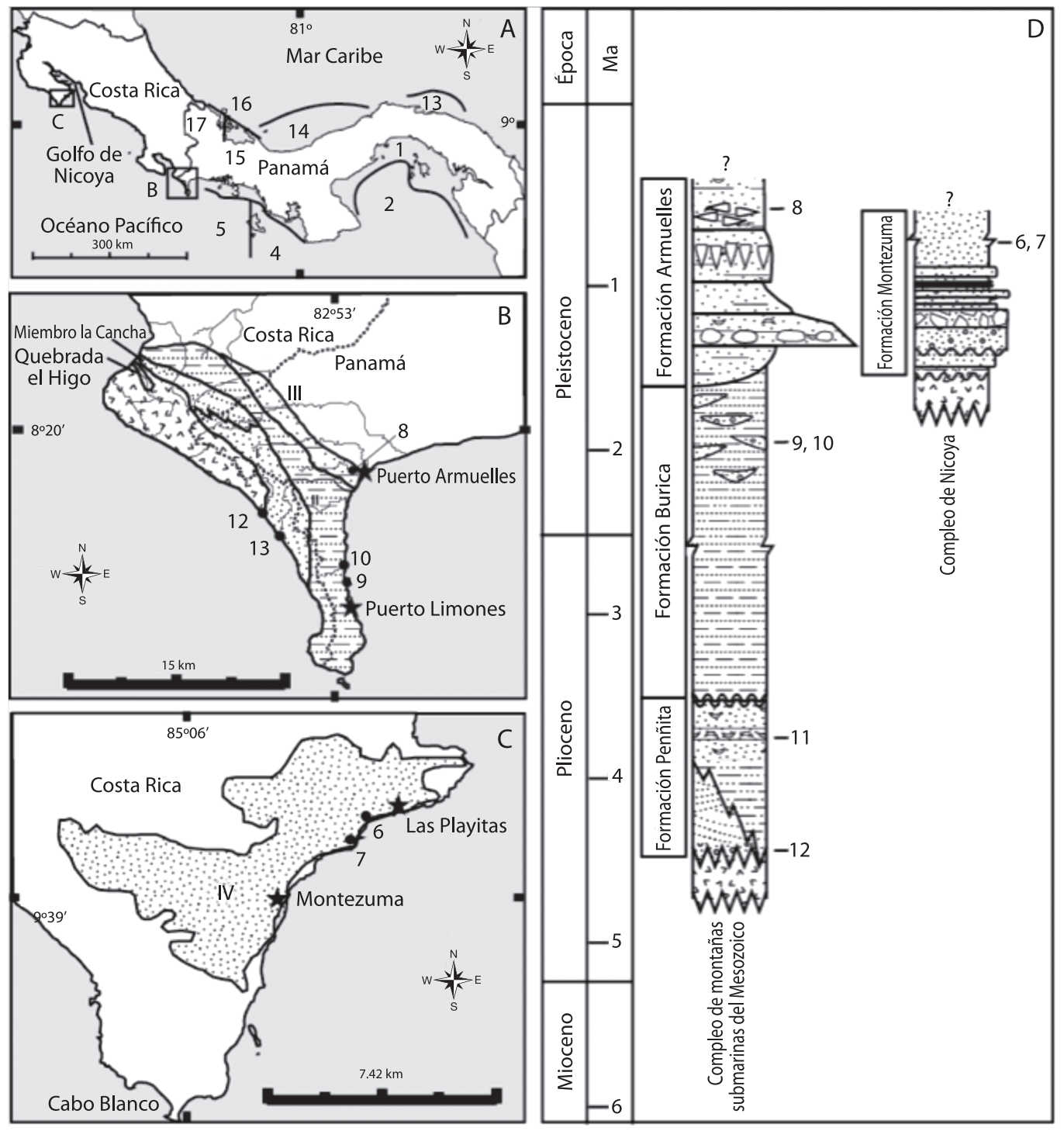

Fig. 2. (A) Los faunulas del Reciente en ambas costas del Istmo de Panamá se marcan con líneas, los recuadros muestran la ubicación de faunulas fósiles en las penínsulas de Nicoya y Burica. (B) Ampliación de la Península Burica con la ubicación de las localidades estudiadas. (C) Ampliación de la parte sureste de la Península Nicoya donde se ubican las localidades de la Formación Montezuma. (D) Columnas estratigráficas de las formaciones geológicas estudiadas. Formación Peñita (I), Formación Burica (II), Formación Armuelles (III), Formación Montezuma (IV). Los números de las faunulas corresponden a los descritos en el cuadro 1. Referencias: Chinchilla-Chavez 1989, Coates et al. 1992, Coates \& Jackson 1998. Las edades han sido ajustadas según Walker \& Geissman (2009) y Gibbard et al. (2010).

Fig. 2. (A) Recent faunules on both coasts of the Isthmus of Panama are marked with lines; boxes show the location of Burica and Nicoya peninsulas. (B) Expansion of Burica Peninsula showing the location of the fossil faunules. (C) Expansion of the southeastern part of the Nicoya Peninsula showing faunules of Montezuma Formation. (D) Lithostratigraphic correlation charts of the geologic formations studied. Peñita Formation (I), Burica Formation (II), Armuelles Formation (III), Montezuma Formation (IV). The number of faunules correspondant to the numbers in the table 1. References: ChinchillaChávez 1989, Coates et al. 1992, Coates \& Jackson 1998. The ages has been adjusted according to Walker \& Geissman (2009) and Gibbard et al. (2010). 
infrayace la Formación Peñita y la suprayace la Formación Armuelles (Fig. 2D) (Coates et al. 1992). Las exposiciones de la Formación Burica se encuentran en una impresionante sección de $20 \mathrm{~km}$ en el lado este de la Península Burica (Fig. 2B). Los sitios ricos en macrofósiles pertenecen al miembro superior (O'Dea et al. 2007b) y se encuentran en la desembocadura de la quebrada Corotú (8 $8^{\circ} 7.821^{\prime} \mathrm{N}$ - 82 $2^{\circ} 52.292^{\prime}$ W) y en la Quebrada Calabazo ( $8^{\circ} 8.777^{\prime}$ $\mathrm{N}-82^{\circ} 52.497$ ' W) (Fig. 2B) donde la fauna es netamente de aguas someras (De Gracia 2009). En estas localidades se encuentran los gasterópodos Anthilophos gaudens, Prunum woldriingei, Cosmioconcha redheri, Phos aff. fusoides y Turritella sp. y, los bivalvos Tellina sp. Nucula sp. Laevicardium sp., Lyrocardium sp., Mactra sp., Saccella cf. phlyctaena, Americardia sp., Barbatia sp., Chione sp., así como fragmentos de Pinna sp. Adicionalmente son encontrados corales ahermatípicos fragmentos y concreciones esféricas de algas calcáreas y, ocasionalmente el gasterópodo planctónico Cavolinia sp. (De Gracia 2009).

La Formación Armuelles tiene un espesor aproximado de $370 \mathrm{~m}$ y data del Pleistoceno temprano-medio (Corringan et al. 1990, Coates et al. 1992). La localidad más importante se encuentra a lo largo del río Rabo de Puerco, iniciando a partir de la carretera en el pueblo de Puerto Armuelles (Fig. 2B) ( $8^{\circ} 16.801^{\prime} \mathrm{N}$ $82^{\circ} 51.995^{\prime} \mathrm{W}$ ) y recorriendo el río en dirección oeste hacia el noreste por $3 \mathrm{~km}$ (Terry 1941). En esta sección, se reconocen cinco facies claramente definidas, la inferior es una capa de limonitas que hace contacto con la Formación Burica (Fig. 2D) (Coates \& Jackson 1998). Luego de la capa del contacto, suprayace una capa de arcillitas de color gris azulado y limolitas con abundantes ichnofósiles. En esta facie se encuentra un horizonte muy característico de peñascos incrustados de ostras, briozoarios incrustantres, corales ahermatípicos y bivalvos donde Tellina sp. es común (Fig. 2D). A esta facie le suprayace una capa de arcillitas azul grisáceas con limonitas sin macrofósiles (Fig. 2D). En la tercera capa, encontramos areniscas limosas de color azul grisáceos con una sorprendente aglomeración de bivalvos Pinna sp. de gran tamaño y en posición de vida, que se extiende horizontalmente por $3 \mathrm{~km}$ (Fig. 2D). La facie superior consiste en sedimentos volcaniclásticos, limolitas lodosas y areniscas con abundantes y diversos moluscos, principalmente de Pinna sp. dispersas pero toda la capa (Fig. 2D) (Coates \& Jackson 1998). La fauna de las facies fosilíferas indica que la Formación Armuelles se depositó en aguas someras a menos de $20 \mathrm{~m}$ de profundidad (De Gracia 2009). A la Formación Armuelles le infrayace la Formación Burica donde el contacto se encuentra en el Río rabo de Puerco, siguiendo el cauce del río por la ruta que siguió Terry (1941) por unos $3 \mathrm{~km}$, en este lugar se pueden observar los sedimentos de la Formación Armuelles encima de las turbiditas de la Formación Burica (Fig. 2D).

La Formación Montezuma se localiza en la península de Nicoya (Fig. 2 C-D). Tiene aproximadamente $30 \mathrm{~m}$ de espesor y es de edad Pleistoceno temprano-medio (Fig. 2 C-D) (Aguilar \& Fischer 1986, Coates et al. 1992). La Formación Montezuma es altamente variable y su localidad tipo está situada a lo largo del camino inmediatamente al oeste del pueblo de Montezuma (Chinchilla-Chaves 1989, Coates et al. 1992). Le infrayacen rocas volcánicas del Complejo de Nicoya y la suprayacen arenas, aluviones y otros sedimentos recientes (Chinchilla-Chaves 1989). Está constituida principalmente por tres litofacies que representan cada una, un ambiente definido: facies de ambiente marino marginal abierto, ensenadas y ambientes marginales protegidos $\mathrm{y}$, ambientes estuarinos-fluviales (Chinchilla-Chaves 1989, Coates et al. 1992). Las muestras colectadas provienen de un estrato altamente fosilífero que aflora en la capa de arenisca a lo largo de la playa Cocalito, ubicada al sur de la Península Nicoya, al norte del pueblo de Montezuma (Fig. 2 C-D).

Muestreo: Las localidades de origen de las muestras fueron separados en unidades aquí denominadas faunulas (O'Dea et al. 2007b) tomadas de las formaciones Armuelles, Burica, 
Montezuma y Peñita (Fig. 1 A-C). Los datos sobre la fauna fósil fueron obtenidos a partir de muestras geológicas de bultos $(n=25)$, recolectadas en faunulas ubicadas en la península de Burica y Nicoya (Cuadro 1, Fig. 2 B-C). En el laboratorio, $170 \mathrm{~kg}$ de sedimentos contenidos en los 25 bultos fueron remojados en agua y puestos en una solución de sal de Glouber para facilitar la extracción de los fósiles (O'Dea et al. 2007b, Surlyk 1972). Posteriormente, el sedimento fue tamizado con cernidores de $2 \mathrm{~mm}$ y todos los fósiles completos o fragmentarios separados en grupos taxonómicos.

Los datos sobre la fauna bentónica reciente se originaron a partir de 174 muestras recolectadas por O'Dea et al. (2007a) con el uso de rastras de fondo y de dragas en la plataforma continental del Caribe de Panamá, así como las 61 muestras recolectadas durante este estudio en la costa Pacífica del Istmo de Panamá (Fig. 2A). Todas las recolectas se hicieron a bordo del R/V Urraca de STRI. Las muestras fueron lavadas en la cubierta del barco y tamizadas con cernidores de $2 \mathrm{~mm}$ y $500 \mu \mathrm{m}$. Los fragmentos de los organismos fósiles y recientes fueron extraídos de la fracción de $2 \mathrm{~mm}$ de las muestras y agrupados en las siguientes categorías: bivalvos, gasterópodos, briozoarios, corales, algas calcáreas (Coralina, Halimeda) y otros (equinodermos, crustáceos, cirrípedios, escafópodos, huesos de peces y otolitos). Los grupos fueron pesados para obtener la abundancia relativa (Cuadro 1) por faunula y la biomasa de cada grupo para reconstruir las comunidades.

Tratamiento de los datos: Los datos en peso de cada muestra reciente y fósil fueron promediados por localidad y luego convertidos a proporciones (Cuadro 1) (que representan la abundancia relativa de cada taxa) como paso previo al análisis de componentes principales (PCA). La abundancia relativa de cada grupo taxonómico en este estudio determina las condiciones ambientales (O’Dea et al. 2007a). Siguiendo este método se hicieron tres análisis de PCA con el programa estadístico Canoco for Windowns (Lepŝ \& Ŝmilauer 2003) y logramos su ordenación con la cual reconstruimos la estructura ecológica de las localidades fósiles mediante su comparación con las localidades recientes. El primer PCA se aplicó para mostrar estadísticamente las diferencias entre faunulas del Pacífico y del Caribe. En el segundo PCA, usamos solo datos de fósiles para reconstruir los ecosistemas del Pacífico y determinar cuáles son las variables de mayor peso. El tercer PCA se aplicó para comparar las faunulas fósiles con las recientes. Esto favorece el correcto análisis del segundo PCA y permite una comparación directa que nos ayude a estimar las condiciones ambientales. De esta manera reconstruimos las comunidades fósiles con la metodología de O’Dea et al. (2007a), las mismas que han sido estandarizadas por el Proyecto de Paleontología de Panamá desde 1986 (Collins \& Coates 1999). Adicionalmente, la información obtenida a partir de los fósiles fue relacionada con los eventos geológicos y ambientales que se dieron durante la etapa final del cierre del Istmo de Panamá en la costa del Pacífico (Shipley \& Moore 1986, Coates et al. 1992, Collins et al. 1995, Kolarsky et al. 1995, Coates \& Obando 1996, Coates 1997, Haug \& Tiedeman 1998, Coates \& Jackson 1998, LeónRodríguez \& Collins 2005, León-Rodíguez 2007, Kamikuri et al. 2009) para obtener conclusiones más precisas.

\section{RESULTADOS}

Fauna reciente: El primer PCA muestra una separación entre faunulas del Pacífico y el Caribe (Fig. 3A). Las comunidades bentónicas del Caribe presentan una estructura ecológica dominada por corales y algas calcáreas. Mientras que comunidades del Pacífico presentan una estructura ecológica dominada por moluscos (Fig. 3A). El resultado de este análisis explica en los tres primeros componentes principales el $89.4 \%$ de la variación total de los datos y una amplia separación entre las faunulas del Caribe y del Pacífico (Fig. 3A). La separación corresponde a diferencias en proporciones de las variables corales y algas con respecto a las variables gasterópodos y bivalvos (Fig. 3A). En la Fig. 3A el punto cinco 


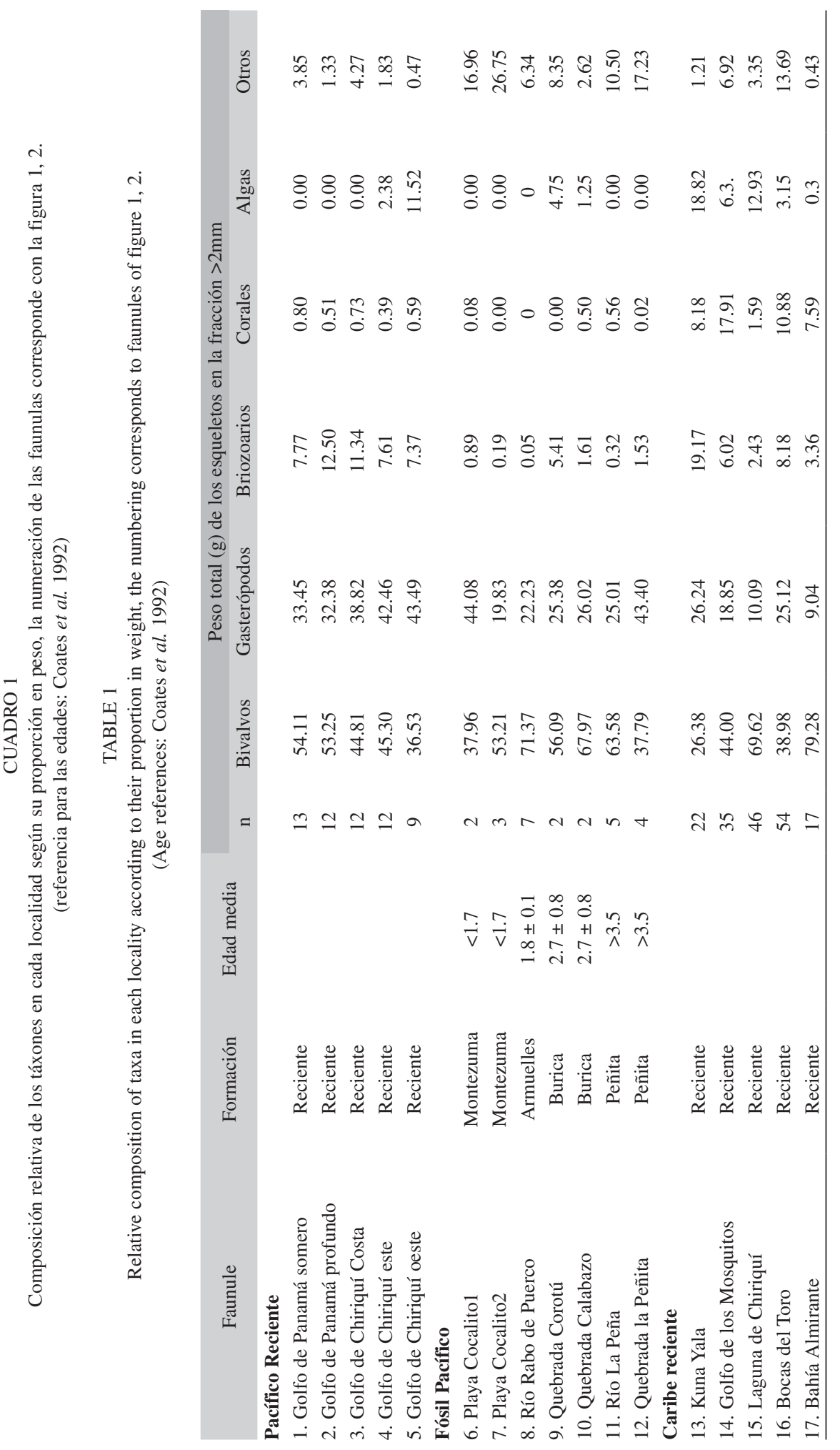



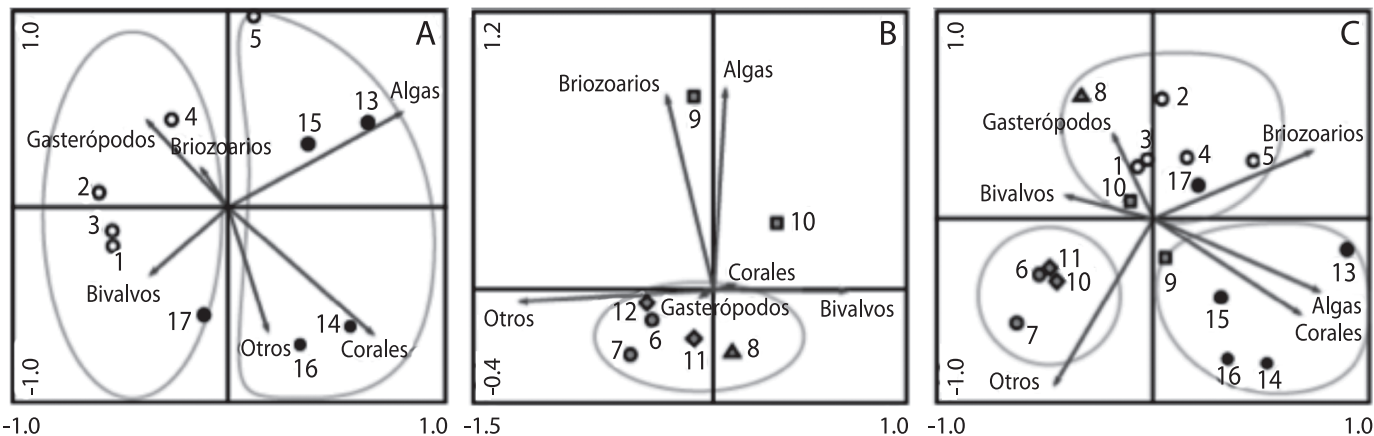

Fig. 3. Análisis de componentes principales construidos con los promedios de los valores de abundancia relativa de fauna según la localidad. (A) Primer PCA donde se analizó la abundancia relativa de fauna Reciente. (B) Segundo PCA donde se muestra el resultado de los análisis de datos fósiles. (C) Tercer PCA donde se muestra el resultado del análisis de todos los datos utilizados en este estudio. Círculos negros: localidades del Caribe, círculos blancos: localidades del Pacífico, rombos grises: Formación Peñita, cuadrados grices: Formación Burica, Triángulos grises: Formación Armuelles, círculos grises: Formación Montezuma. Los números coinciden con la figura 1 y el cuadro 1.

Fig. 3. PCA analysis constructed with the averages values of relative abundance of fauna according faunules. (A) First PCA showing the analysis of the relative abundance of Recent fauna. (B) Second PCA showing the results of the analysis of fossil data which represent. (C) Third PCA showing the result of analysis of all data used in this study. Black circles: Caribbean localities, white circles: pacific localities, grays rhombuses: Peñita Formation, grays squares: Burica Formation, Grays triangles: Armuelles Formation, grays Montezuma Formation. The numbers correspond to figure 1 and table 1.

que corresponde al Golfo de Chiriquí Oeste (Pacífico) se agrupa con faunulas del Caribe (Fig. 3A). Por otra parte, también se observa que el punto 17 que corresponde a la Bahía de Almirante (Caribe), se agrupa con faunulas del Pacífico y que están dirigidas por la variable de bivalvos (Fig. 3A).

Fauna fósil: El segundo PCA explica en los dos primeros componentes principales el $91.13 \%$ de la variación de los datos. Las faunulas Quebrada Corotú y Quebrada Calabazo (punto 9 y 10 Fig. 3B), de la Formación Burica ( 2.7Ma), no se agrupan con el resto de faunulas fósiles. Adicionalmente, la fauna del río Rabo de Puerco (punto 8 Fig. $3 \mathrm{~B})$ de la Formación Armuelles ( 1.8Ma), se encuentra controlada por la variable bivalvos mientras que, el resto de las localidades, las cuales corresponden a la Formación Peñita $(>3.5 \mathrm{Ma})$ y Montezuma $(<1.7 \mathrm{Ma})$ están dirigidas hacia la variable otros (Fig. 3B). Esta última variable corresponde mayormente a cirrípedos de zonas intermareales (Cuadro 1) (De Gracia 2009).
En el tercer PCA (Fig. 3C) se analizaron todas las faunulas estudiadas. La prueba explica el $85.7 \%$ de la varianza total en los tres primeros componentes principales. La gráfica (Fig. 3C) muestra que los tres grupos de análisis (Caribe Reciente, Pacífico Reciente y Pacífico fósil), están distribuidos casi en grupos separados (Fig. 3C) donde faunulas del Pacífico (fósil y reciente) están más relacionadas (Fig. 3C). En el análisis esto se debe a que la variable 'otros' dirige la ecología en las faunulas de la Formación Peñita y de la Formación Montezuma (Fig. 3C). Adicionalmente, la faunulas de la Formación Burica, Quebrada Corotú (punto 9 Fig. 3C) se relaciona con faunulas del Caribe y la faunula Quebrada Calabazo (punto 10 Fig. 3C), se relaciona más con localidades del Pacífico. Esto indica ambientes variables de aguas someras para la parte superior de la formación Burica. Las condiciones de cada faunula fueron reconstruidas a partir de los análisis de PCA, usando estudios taxonómicos de la fauna marina y correlacionando estos datos con los principales eventos geológicos y ecológicos que se dieron en el pasado (Fig. 4). 


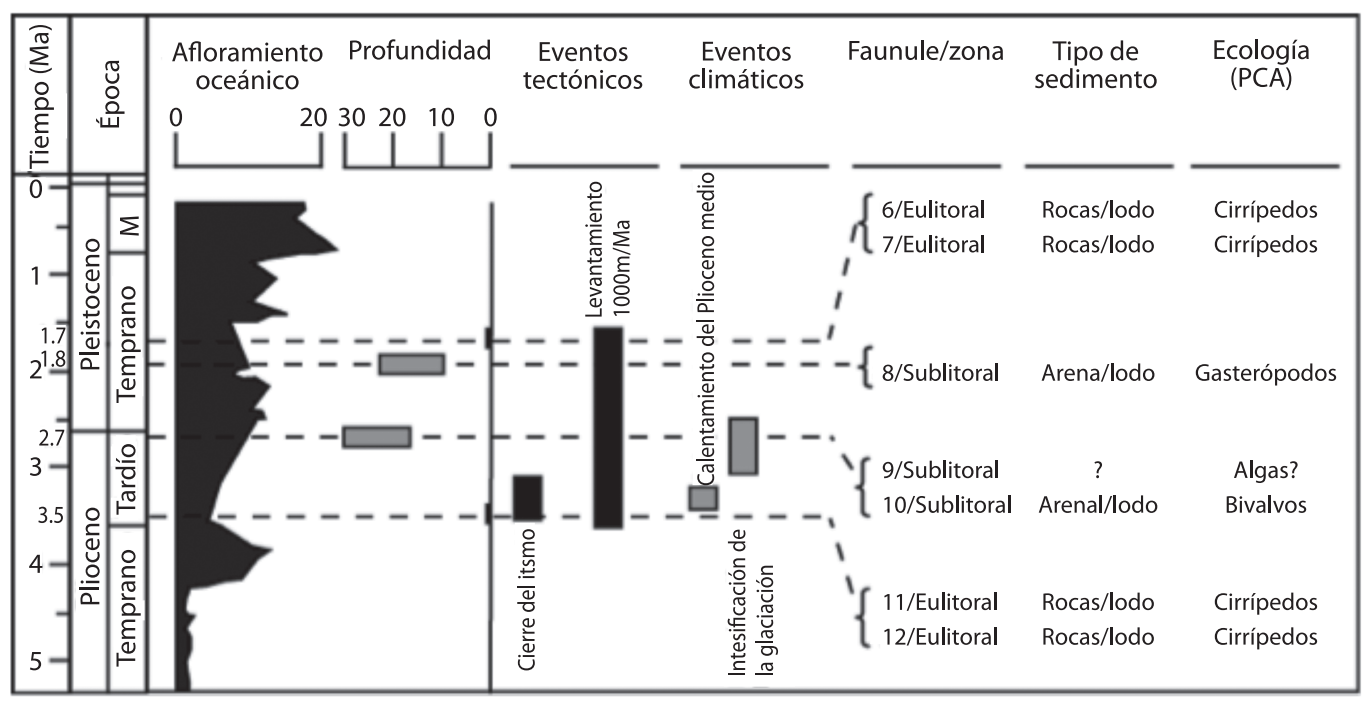

Fig. 4. Reconstrucción ambiental de las faunulas estudiadas y su correlación con estimaciones de afloramiento oceánico (Kamikuri et al. 2009), Paleobatimetría (este estudio), eventos tectónicos (Coates et al. 1992, Coates \& Obando 1996, Collins et al. 1995, Coates 1997) y eventos climáticos (Haug \& Tiedeman 1998). La imagen muestra los cambios locales que se dieron en las faunulas de la Península Burica y la reconstrucción de las faunulas de la Península Nicoya. El número de las faunulas es igual a lo indicado en el cuadro 1. Las edades han sido ajustadas según Walker \& Geissman (2009) y Gibbard et al. (2010).

Fig. 4. Environmental reconstruction of the fossils faunules and the correlation of this whit estimates of ocean upwelling (Kamikuri et al. 2009), Paleobathymetry (this study), tectonic events (Coates et al. 1992, Coates \& Obando 1996, Collins et al. 1995, Coates 1997) and climatic events (Haug \& Tiedeman 1998). The image shows the local changes that occurred in the faunules of Burica Peninsula and the reconstruction of the Nicoya Peninsula faunules. The faunules numbers match with figure 1,2 and table 1.

\section{DISCUSIÓN}

Comunidades modernas en el Pacífico: Los resultados presentados coinciden con estudios que indican que las condiciones oceanográficas tienen fuerte influencia sobre la fauna bentónica y que las comunidades entre el Mar Caribe y el POT son diferentes (D'Croz \& Robertson 1997, Jackson \& D'Croz 1997, O'Dea et al. 2007a). El estudio de O'Dea et al. (2007a), explica que hay una relación inversa entre las variables bivalvos y gasterópodos con respecto a la variable corales y algas. También, que la abundancia de bivalvos y gasterópodos se relaciona con alta productividad planctónica en zonas de afloramiento. Mientras que la dominancia de corales y algas generalmente está asociada a ecosistemas sin exposición a las aguas de afloramiento.
La agrupación de la faunula del Golfo de Chiriquí Oeste con faunulas del Caribe la asociamos con la ausencia de afloramiento en el Golfo de Chiriquí y a la presencia del arrecife de coral en la Isla de Coiba, que es el mayor arrecife costero del POT (Guzmán \& Cortes 1993, Glynn \& Maté 1997, Maté 2003). Por otra parte, la agrupación de la faunula Bahía Almirante con faunulas del Pacífico en el primer PCA, indica que las comunidades son dominadas por bivalvos. Este resultado es explicado por el efecto de la escorrentía continental de la ciudad de Almirante que afecta las áreas costeras en esta zona (D'Croz et al. 2005). La descarga de nutrientes por escorrentía a causa de la deforestación y por la ciudad de Almirante (D'Croz et al. 2005) favorecen el desarrollo de táxones filtradores como bivalvos lo que es indicativo de un cambio en las 
comunidades causado por el impacto humano. La estructura ecológica de la costa del Golfo de Chiriquí en el primer PCA, es similar a la de las zonas de afloramiento del Golfo de Panamá. Sin embargo, la disponibilidad de nutrientes en este faunula no está asociada con un afloramiento de tipo eólico (D'Croz \& O'Dea 2007, 2009) aunque un incremento de la fertilidad en aguas superficiales podría estar relacionada con el efecto de ondas internas de agua en el Golfo de Chiriquí (D’Croz \& O’Dea 2009) y a la descarga de sedimentos de los ríos, (D'Croz \& O’Dea 2007). En adición, los extensos bosques de manglar que cubren la costa en el Golfo de Chiriquí (D’Croz 1993) también favorecen el desarrollo de comunidades dominadas por bivalvos, ya que aportan nutrientes en suspensión y sustratos lodosos. Por otra parte, el resto de las faunulas del Golfo de Chiriquí presentan condiciones más favorables para el desarrollo de arrecifes principalmente porque la temperatura del agua es más cálida y hay pocos nutrientes disueltos (D’Croz \& O’Dea 2007, 2009). En esta zona el afloramiento eólico está ausente y esto se atribuye a la presencia de la cordillera que bloquea el paso de los vientos Alisios entre el Valle de Antón en Panamá hasta el Volcán Arenal en Costa Rica (Dana 1975, Kwiecinski \& Chial 1983).

Ambientes del pasado en Burica y Nicoya: Mediante el análisis de PCA y el estudio taxonómico de la fauna presente en los faunules (De Gracia 2009) fue posible reconstruir la estructura ecológica de las comunidades fósiles en la Península Burica y Nicoya. La presencia de corales y algas calcáreas en la Formación Burica representa una diferencia importante en estas faunulas. Las conchas de los bivalvos en estos lugares son poco armadas y ornamentadas, morfologías muy similares a las conchas típicas del Caribe, como ejemplos de este tipo de conchas para esta faunula tenemos: Tellina sp., Laevicardium sp., Mactra sp. y Prunum woldriingei (Olsson 1942, O'Dea et al. 2007b). Ecológicamente, conchas de arquitectura como esta reflejan una baja incidencia de depredación (Jackson \& D’Croz 1997). En faunulas con fósiles de la Formación Burica se han encontrado numerosos fragmentos de corales, algas calcáreas y concreciones esféricas de algas calcáreas (Coates et al. 1992) que indican aguas con poca turbidez. Esta observación coincide con el resultado del tercer PCA, donde la faunula de Quebrada Corotú (faunula 9) se asocia con localidades del Caribe. Para la faunula de Quebrada Calabazo, no se da esta situación y estimamos un ambiente con sustrato arenoso-lodoso ubicado en la zona sublitoral. El segundo PCA también muestra que la estructura de las comunidades en la Formación Armuelles se encuentra dominada por bivalvos. En la faunula Rabo de Puerco, encontramos agregaciones extensas de grandes conchas de Pinna sp. ubicadas in situ (Fig. 2D) (Olsson 1942, Coates \& Jackson 1998, O’Dea et al. 2007b), asociadas a Ostrea sp. (Olsson 1942, De Gracia 2009), lo que sugiere una alta disponibilidad de nutrientes y sustratos arenoso - lodoso en la zona sublitoral. A los niveles de Pinna sp. in situ le suprayace otro nivel portador de los mismos moluscos pero dispersos y quebrados. Esta evidencia y los sedimentos volcaniclásticos indican que un evento súbito sepultó in situ las agregaciones de Pinna sp. en un momento único.

Según el segundo PCA en la Formación Peñita y Montezuma la variable "otros" domina la ecología. Esta variable corresponde casi en su totalidad a cirrípedos que son organismos filtradores y que pertenecen a la zona eulitoral (De Gracia 2009). Para la Formación Peñita las asociaciones de fauna (Olsson 1942, Aguilar 1987, O’Dea et al. 2007b) están constituidas por erizos Eucidaris sp., y moluscos como Anadara sp., Strombina sp. y Crepidula sp., ellas sugieren ambientes con buena disponibilidad de nutrientes en sustratos rocoso-lodosos en la zona eulitoral. En la Formación Montezuma, los cirrípedos encontrados pertenecen al género Tetraclita (De Gracia 2009), que actualmente habitan en zonas intermareales sobre costas rocosas (Laguna 1985). Cirrípedos de este género, Tetraclita panamensis (Newman \& Ross 1976, Laguna 1985) se encuentran en el Pacífico formando comunidades con 
numerosos individuos en zonas de afloramiento en Panamá y Costa Rica (Sibaja-Cordero \& Cortés 2008). Las asociaciones de fauna que encontramos en las faunulas de la Formación Montezuma (De Gracia 2009) coinciden con la reportada por Aguilar \& Fisher (1986). Por lo cual se sugerimos para los faunules de playa Cocalito en la Formación Montezuma, ambientes estuarinos y bahías con influencia oceánica muy productivas.

Con el tercer PCA se establecen relaciones entre las comunidades fósiles y recientes. Los fósiles se relacionan con faunulas del Pacífico pero no se agrupan totalmente con estos ya que la ecología en las formaciones Peñita y Montezuma están determinadas por la presencia de cirrípedos de zonas intermareales (De Gracia 2009). El efecto de separación entre la fauna fósil y la reciente del Pacífico, se atribuye a la variación en las paleoprofundidades de faunulas de las formaciones Peñita y Montezuma, ya que estas pertenecen a la zona eulitoral, pero las faunulas recientes van desde aguas someras hasta $250 \mathrm{~m}$ de profundidad en el Golfo de Panamá. La faunula Quebrada Corotú se agrupa cercanamente a localidades del Caribe en el tercer PCA. Esto se debe a que las algas (bolas de algas) causan un efecto importante sobre la ecología de esta faunula. Los sedimentos del miembro inferior de la Formación Burica corresponden a aguas abisales entre 2 000m y 2 400m (Corringan et al. 1990, Coates et al. 1992). La Quebrada Corotú se ubica en el miembro superior de Burica donde la paleoprofundidad decrece drásticamente, esto indica que esta faunula se depositó en lugares alrededor de islas pequeñas que crearon condiciones protegidas favorables para el desarrollo de las algas calcáreas y corales como sucede en la parte inferior de la Formación Peñita (Coates et al. 1992) y en la Península de Osa (Kriz 1990). Sin embargo, al hallar estas algas solo en la Formación Burica nos hace concluir que estas condiciones fueron momentáneas y que, posteriormente desaparecieron dando paso nuevamente a ambientes de afloramiento como lo indican estimaciones de temperaturas costeras (Teranes et al. 1996) y oceánicas
(Kamikuri et al. 2009) y, datos de fauna bentónica (De Gracia 2009).

Alta productividad en el pasado: Las asociaciones de fauna de moluscos (datos no publicados) y peces para la Formación Curré del Pacífico se correlacionan con la fauna de la Formación Gatún del Caribe (Laurito \& Valerio 2008, Aguilar et al. 2010). Esto sugiere ambientes de aguas turbias y con nutrientes para la región de Burica durante el Mioceno, época en que el Istmo de Panamá no había completado su formación y el Pacífico estaba conectado con el Caribe. Durante el Plioceno temprano el afloramiento había comenzado a declinar y disminuía en intensidad dramáticamente. (O'Dea et al. 2007a). Del mismo modo en el Pacífico, estimaciones con isótopos y asociaciones de fauna indican que lo mismo ocurrió en zonas costeras y oceánicas (Teranes et al. 1996, Kameo 2002, Kamikuri et al. 2009). Los sedimentos de la parte inferior de la Formación Burica fueron depositados en aguas de aproximadamente $2300 \mathrm{~m}$ (Coates et al. 1992). Esta profundidad decrece gradualmente y para el Plioceno Tardío (2.7Ma), la fauna encontrada en faunulas de la parte superior de la Formación Burica no se asocia a lugares con afloramiento. Las algas calcáreas, bolas de algas y corales ahermátipicos de aguas someras presentes en estas faunulas no corresponden a ambientes de afloramiento (Coates et al. 1992, De Gracia 2009). En contraste, a esta evidencia para aguas costeras, los datos oceánicos indican incrementos del afloramiento en mar abierto durante las mismas fechas (Kamikuri et al. 2009). Debido a las variaciones del basamento en la DAC, pensamos que el miembro superior de la Formación Burica se depositó en una especie de archipiélago que protegía las aguas de las condiciones oceánicas y de afloramiento $\mathrm{y}$, que este se unió gradualmente a medida que la DAC hacia subducción. Investigaciones previas indican que también existieron islas en la zona de Península de Osa en Costa Rica (Berrangé 1989, Kriz 1990) y que a causa de la deformación causada por el Choque de la DAC en la región de Burica estas desaparecieron 
gradualmente y formaron el Golfo Dulce y la Península de Osa (Corringan et al. 1990). Los datos geológicos indican que la disminución del nivel del mar desde aguas abisales hasta aguas someras en sedimentos de la formación Burica ocurrió en tan solo 1Ma. (Coates et al. 1992). A causa de este efecto, los ambientes protegidos en estas costas permanecieron por un corto tiempo y desaparecieron mientras continuaba el levantamiento del lecho marino. Análisis de isotopos de oxígeno en conchas de moluscos de la Formación Armuelles, indican que las temperaturas de afloramiento se restablecieron para el Pleistoceno temprano en Burica (Teranes et al. 1996). La evidencia de las asociaciones de fauna apoya esta idea ya que en la Formación Armuelles son abundantes moluscos como Pinna sp., Ostrea sp. y Pecten sp. que son típicos de zonas de afloramiento con alta disponibilidad de nutrientes (Olsson 1942, O'Dea et al. 2007b). Reforzando este argumento, las estimaciones de surgencias en mar abierto para el Pacífico también indican un afloramiento intenso en las fechas en que se depositaron los sedimentos de la Formación Armuelles (Kamikuri et al. 2009).

Los estratos de Pinna sp. in situ sepultados bajo el estrato fosilífero de Pinna sp. fragmentada y dispersa son indicativos de una alta actividad tectónica y un evento catastrófico que ocurrió durante el Pleistoceno medio. Los datos geológicos indican que las velocidades de levantamiento en el Pleistoceno medio fueron mucho más altas de lo que se creía (de 8m/1 000años) (León-Rodríguez \& Collins 2005, León-Rodríguez 2007). Esta es evidencia apoya nuestra idea ya que en el Río Rabo de Puerco, la deposición de la Formación Armuelles culmina con el estrato de sedimentos de limonitas volcanoclásticas lodosas mezclada con areniscas, lo que revela actividad volcánica en las zonas adyacentes (Coates \& Jackson 1998). La abundante fauna de moluscos transportados y los múltiples fragmentos de Pinna sp. dispersos y que suprayacen sobre el nivel de Pinna sp. in situ indican que esta facie fue sepultada en un momento único.
En la Península Nicoya, comunidades del Pleistoceno temprano también sugieren un afloramiento intenso. El indicador más claro es la presencia de cirrípedos intermareales del genero Tetraclita, estos se encuentran habitando zonas de afloramiento como el Golfo de Panamá (De Gracia 2009). Actualmente, en el Golfo de Nicoya existe afloramiento eólico y mucha escorrentía de los ríos que mantienen una alta disponibilidad de nutrientes (Brenes et al. 2003). Esto quiere decir que la alta disponibilidad de nutrientes ha persistido en Nicoya. Sin embargo, pensamos que los cambios geológicos producto del levantamiento, causaron la disminución del afloramiento en la mayor parte de lo que hoy es la península Nicoya y que, las zonas alta disponibilidad de nutrientes, que en el pasado presentaron una mayor extensión en Costa Rica, han quedado restringidas al Golfo de Nicoya y al Golfo de Papagayo. En Nicoya, los efectos de la subducción no son tan pronunciados como ocurre en la península Burica y Osa (Corringan et al. 1990, Kolarsky et al. 1995). Esto es así debido a que el ángulo de subducción en Nicoya es de $\sim 7^{\circ}$ en comparación con Burica donde el ángulo es casi nulo (Shipley \& Moore 1986) haciendo que el levantamiento topográfico en Nicoya se diera en menor escala.

Actualmente, las montañas que impiden el paso de los vientos Alisios permiten el desarrollo de los mejores arrecifes de coral en el POT porque no ocurre afloramiento (Glynn et al. 1972, Cortés 1997, Cortés \& Jiménez 2003, Maté 2003). Sin embargo, estas condiciones son de origen reciente ya que datos de afloramiento oceánico, isótopos y asociaciones de fauna indican que para el Pleistoceno existían surgencias en la región (Teranes et al. 1996, Kamikuri et al. 2009, De Gracia 2009). El rápido levantamiento topográfico de la cordillera que inició en el Plioceno superior con velocidades de $1000 \mathrm{~m} / \mathrm{Ma}$ (entre 3.6-1.6Ma) levantó el lecho marino hasta aguas someras (Coates et al. 1992). Durante el Pleistoceno medio, un dramático incremento en las velocidades con un índice de levantamiento de 8m/1000 años (León-Rodríguez \& Collins 2005, León-Rodríguez 2007) causó la elevación de la Cordillera de Talamanca. Esto causó 
que los ambientes de Afloramiento reportados en las Formaciones Armuelles y Montezuma, desaparecieran permitiendo el desarrollo de los arrecifes de coral más emblemáticos del POT (Glynn et al. 1972, Cortés 1997, Cortés \& Jiménez 2003, Maté 2003).

Los procesos geológicos ocurridos en la Península Burica y Nicoya fueron complejos y determinaron las condiciones ambientales que se dan hoy en las costas del POT que abarcan desde el Valle de Antón en Panamá hasta el Arenal en Costa Rica. Las comunidades fósiles del Plioceno temprano de la formación Peñita, indican surgencias marino costeras y que los sedimentos se depositaron alrededor de islas oceánicas. Durante el Plioceno tardío y el Pleistoceno temprano, hubo un gran declive de la profundidad del océano debido al levantamiento donde aguas abisales de más de $2300 \mathrm{~m}$ de profundidad, pasaron a ser aguas poco profundas de no más de $40 \mathrm{~m}$. Las variaciones en al basamento de la DAC crearon islas en zonas costeras que mantenían resguardada la zona de la influencia oceánica y del afloramiento que ocurría intensamente en mar abierto. Esta condición solo persistió durante corto tiempo, ya que el levantamiento tectónico continuaba y las islas se unieron a tierra firme. Para el Pleistoceno Temprano, el afloramiento regresó a las aguas costeras como lo indican los fósiles de la Formación Armuelles, Montezuma y la evidencia en investigaciones previas.

El afloramiento era posible debido a que la cordillera de Talamanca no se había elevado y los vientos alisos podían cruzar libremente. El levantamiento de la cordillera ocurrió muy recientemente (durante el Pleistoceno) con velocidades de $8 \mathrm{~m} / 1$ 000años entre 0.46$0.26 \mathrm{Ma}$. Esta intensa actividad geológica provocó la elevación de la cordillera de Talamanca a su altura actual. Las montañas bloquearon el paso de los vientos Alisios entre las regiones que abarcan desde el Valle de Antón hasta el Arenal en Costa Rica y el afloramiento quedó restringido a zonas donde las montañas son bajas como el Golfo de Panamá, Golfo de Nicoya y Golfo de Papagayo. La cordillera bloqueó el paso de los vientos Alisios impidiendo las surgencias costeras en la región estudiada y creó condiciones favorables para el desarrollo de los arrecifes costeros de coral más grandes del POT. De esta manera concluimos que la subducción de la DAC provocó importantes cambios que modificaron las condiciones ambientales y la estructura ecológica entre Panamá y Costa Rica.

\section{AGRADECIMIENTOS}

Agradecemos a Anthony Coates por ayudarnos con la geología de las áreas de estudio. A Jeremy B. C. Jackson por permitir la realización de esta investigación en los laboratorios de STRI. A Celideth De León y a todo el personal de Recursos Minerales de Panamá por los permisos de recolecta. A Mercedes Arosemena y Marissa Batista de los laboratorios marinos en Naos de STRI por su apoyo logístico durante la fase de campo de esta investigación. A Nélida Gómez de programas académicos de STRI. A nuestro guía de campo Osvaldo. A Jorge Cortés y Ana Fernández de la Universidad de Costa Rica por su valioso apoyo con material bibliográfico. A Steve Paton por la confección de la figura 1. A Luz Cruz de SENACYT por la invitación para asistir al taller de escritura de artículos científicos. A Orangel Aguilera por las revisiones y críticas a las ediciones tempranas de este manuscrito. A Jennifer Keyle, Kraig Kraft y Robert Beatty del Sustainable Sciences Institute por sus revisiones y comentarios para mejorar este artículo. El financiamiento para la realización de esta investigación fue proporcionado por la National Science Foundation (EAR03-45471) y el Smithsonian Marine Science Network para las expediciones marinas donde se obtuvieron las muestras de dragado y arrastres. A la National Geographic Society Exploration Grant por el apoyo para la exploración y recolecta de los fósiles y la beca Abdiel Adames del Smithsonian Tropical Research Institute que permitió el trabajo de laboratorio. Agradecemos el apoyo financiero brindado por el Sistema Nacional de Investigación de la Secretaría Nacional de Ciencia, Tecnología e Innovación de Panamá. 


\section{RESUMEN}

Con el fin de evaluar el efecto de la Subducción de la Dorsal Asísmica de Cocos (DAC) durante las etapas finales de la formación del Istmo. Realizamos muestreos con bultos en afloramientos fosilíferos en las penínsulas de Burica y Nicoya. Las condiciones paleoecológicas y paleoambientales fueron reconstruidas a partir de la comparación entre la estructura de las comunidades fósiles, con las comunidades modernas dragadas de los mares de Panamá, usando análisis de componentes principales. Los resultados indican que antes del cierre del Istmo, existieron islas oceánicas y un afloramiento moderado en Burica. Posterior al cierre, el choque de la DAC provocó la elevación del fondo marino y las aguas que se encontraban a 2 $300 \mathrm{~m}$ pasaron a $40 \mathrm{~m}$. El afloramiento se intensificaba en mar abierto pero la dorsal había formado islas en Burica que limitaban el efecto del afloramiento en la costa. La subducción de la DAC continuó y las islas se unieron gradualmente a tierra firme y desaparecieron, permitiendo el afloramiento. Durante el Pleistoceno medio un segundo proceso de levantamiento acelerado continuó elevando el fondo marino y formó la Cordillera de Talamanca. La cordillera creó una barrera que bloqueó el paso de los vientos Alisios y originó condiciones ecológicas y optimas que permiten el crecimiento de los mejores arrecifes de coral costeros del Pacífico oriental tropical (POT) entre Panamá y Costa Rica.

Palabras clave: cordillera de Talamanca, cierre del Istmo de Panamá, afloramiento, comunidades bentónicas, Océano Pacífico, fósiles marinos.

\section{REFERENCIAS}

Aguilar, T. 1987. Comparación entre la fauna malacológica de las principales formaciones del Plioceno Marino de Costa Rica, América Central. Rev. Geol. Am. Central 6: 43-73.

Aguilar, T., B. Acevedo \& A. Ulloa. 2010. Paleontología de una sección del río Corredores, Formación Curré, Mioceno superior, cantón de Coto Brus, provincia de Puntarenas, Costa Rica. Rev. Geol. Am. Central 42: 43-75.

Aguilar, T. \& R. Fischer. 1986. Moluscos de la Formación Montezuma (Plioceno-Pleistoceno; Costa Rica). Geol. Paleontol. 20: 209-2041.

Alvarado, G.E., C. Dengo, U. Martens, J. Bundschuh, T. Aguilar \& S.B. Bonis. 2007. Stratigraphy and geologic history, p. 345-394. In J. Bundschuh \& G.E. Alvarado (eds.). Central America: Geology, resources and hazzards vol. 2. Taylor \& Francis, Londres, Reino Unido.
Berrangé, J.P. 1989. The Osa group: an auriferus Pliocene sedimentary unit from the Osa Peninsula, Southern Costa Rica. Rev. Geol. Am. Central 10: 67-93.

Brenes, C.L., J.E. Coen, D.B. Chelton, D.B. Enfield, S. León \& D. Ballestero. 2003. Wind driven upwelling in the Gulf of Nicoya, Costa Rica. Int. J. Remote Sens. 24: 1127-1133.

Chinchilla-Chavez, A.L. 1989. Estudio geológico de la Formación Montezuma (Plio-Pleistoceno, Península de Nicoya, Costa Rica). Tesis de Licenciatura, Universidad de Costa Rica, San José, Costa Rica.

Coates, A.G. 1997. The Forging of Central America, p. 1-37. In A.G. Coates (ed.). Central America: A natural and Cultural history. Yale University, New Haven, EEUU.

Coates, A.G., L.S. Collins, M.P. Aubry \& W.A. Berggren. 2004. The geology of the Darien, Panama, and the late Miocene-Pliocene collision of the Panama arc with northwestern South America. Geol. Soc. Am. Bull. 116: 1327-1344.

Coates, A.G. \& J.A. Obando 1996. The geologic evolution of the Central American isthmus, p. 21-56. In J.B.C. Jackson, A.F. Budd \& A.G. Coates (eds.). Evolution and environment in tropical America. Chicago University, Chicago, EEUU.

Coates, A.G. \& J.B.C. Jackson. 1998. Neogene sedimentary basins of Costa Rica and Panama. $11^{\text {th }}$ Conference Inter. Bryozoology Association, Panama. 39 p.

Coates, A.G., J.B.C. Jackson, L.S. Collins, T.M. Cronin, H.J. Dowsett, L.M. Bybell, P. Jung \& J.A. Obando. 1992. Closure of the Isthmus of Panama: The NearShore Marine Record of Costa Rica and Western Panama. Geol. Soc. Am. Bull. 104: 814-828.

Collins, L.S. \& A.G. Coates. 1999. A paleobiotic survey of Caribbean Faunas from Neogene of the Isthmus of Panama. Bull. Am. Paleontol. 357: 5-351.

Collins, L.S., A.G. Coates, J.B.C. Jackson \& J.A. Obando. 1995. Timing and rates of emergence of the Limon and Bocas del Toro Basins: Caribbean effects of Cocos Ridge subduction?, p. 263-290. In P. Mann (ed.). Geologic and tectonic development of the Caribbean plate boundary in southern Central America. Geol. Soc. Am., Special Paper.

Collins, L.S., A.F. Budd \& A.G. Coates 1996. Earliest evolution associated with closure of the Tropical American Seaway. Proc. Natl. Acad. Sci. USA. 93: 6069-6072. 
Corringan, J.D., P. Mann \& J.C. Ingle Jr. 1990. Foreal response to subduction of the Cocos ridge, PanamaCosta Rica. Geol. Soc. Am. Bull. 102: 628-652.

Cortés, J. 1997. Biology and geology of coral reefs of the eastern Pacific. Coral Reefs 16: 39-46.

Cortés, J. \& C. Jiménez. 2003. Coral reefs of the Pacific of Costa Rica, p. 361-375. In J. Cortés (ed.). Latin American Coral Reefs. Elsevier Science, Amsterdam, Holanda.

Dana, T.F. 1975. Development on contemporary eastern Pacific coral reefs. Mar. Biol. 33: 355-374.

D'Croz, L. 1993. Status and uses of mangrove in the Republic of Panama, p. 115-127. In L.D. Lacerda (ed.) Conservation and sustainable utilization of mangrove forests in Latin America and Africa Regions. Part I. ITTO/ISME Mangrove Ecosystems Technical Reports, Okinawa, Japón.

D’Croz, L. \& D.R. Robertson. 1997. Coastal oceanographic conditions affecting coral reefs on both sides of the Isthmus of Panama. Proc. 8th Int. Coral Reef Symp. 2: 2053-2058.

D’Croz, L. \& A. O’Dea 2007. Variability in upwelling along the Pacific shelf of Panama and Implications for the Distribution of Nutrients and Chlorophyll. Estuar. Coast. Shelf S. 73: 325-340.

D’Croz, L. \& A. O’Dea. 2009. Nutrient and chlorophyll dynamics in Pacific Central America (Panama), p. 335-344. In M.A. Lang, I.G. Macintyre \& K. Rützler (eds.). Proceedings of the Smithsonian Marine Science Symposium. Smithsonian Contributions to the Marine Sciences, Smithsonian Institution Scholarly, Washington D.C., EEUU.

D’Croz, L., J. Del Rosario \& P. Góndola. 2005. The effect of fresh water runoff on the distribution of dissolved inorganic nutrients and plankton in the Bocas del Toro Archipelago, Caribbean Panama. Caribb. J. Sci. 41: 414-429.

De Gracia, C. 2009. Estructura de las comunidades bentónicas entre la costa pacífica de Panama y Costa Rica durante la formación del Istmo centroamericano. Tesis de Bachillerato, Universidad de Panamá, Panamá.

Gibbard, P.L., M.J. Head \& M.J.C. Walker. The Subcommision on Quaternary Stratigraphy. 2010. Formal ratification of the Quaternary system/period and the Pleistocene series/epoch whith a base at $2.58 \mathrm{Ma}$. J. Quaternary Sci. 25: 96-102.
Glynn, P.W. 1977. Coral growth in upwelling and nonupwelling areas off the Pacific coast of Panama. J. Mar. Res. 35: 567-585.

Glynn, P.W. \& J.L. Maté 1997. Field guide to the Pacific coral reefs of Panama. Proc. 8th Int. Coral Reef Symp. 1: 145-166.

Glynn, P.W., S.B. Coley, N.J. Gassman, K. Black, J. Cortés \& J.L. Maté. 1996. Reef coral reproduction in the eastern Pacific: Costa Rica, Panama and Galapagos Islands (Ecuador). IV. Agariciidae, recruitment and recovery of Pavona varians and Pavona sp.a. Mar. Biol. 136: 786-805.

Glynn, P.W., R.H. Stewart \& J.E. McCosker. 1972. Pacific coral reefs of Panama: structure, distribution and predators. Geol. Rundsch. 61: 483-519.

Guzmán, H. \& J. Cortés. 1993. Arrecifes coralinos del Pacífico Oriental: revisión y perspectiva. Rev. Biol. Trop. 41: 535-557.

Haug, G. \& R. Tiedemann. 1998. Effects of the formation of the Isthmus of Panama on Atlantic Ocean thermoline circulation. Nature 393: 673-676.

Jackson, J.B.C. 1994. Constancy and change of the life in the sea. Philos. Trans. R. Soc., B. 334: 55-60.

Jackson, J.B.C. \& L. D`Croz, 1997. The Ocean Divided, p. 38-71. In A.G. Coates (ed.). Central America: a Natural and Cultural History. Yale University, New Haven, EEUU.

Jackson, J.B.C., P. Jung, A.G. Coates \& L.S. Collins 1993. Diversity and extinction of tropical American mollusks and emergence of the Isthmus of Panama. Science 260: 1624-1626.

Jackson, J.B.C., P. Jung \& H. Fortunato. 1996. Paciphilina revisited: Transissthmian evolution of the Strombinagroup (Gastropoda:Columbellidae), p. 234-270. In J.B.C. Jackson, A.G. Coates \& A.F. Budd (eds.). Evolution and environment in tropical America. University of Chicago, Chicago, EEUU.

Jain, S. \& L. Collins. 2007. Trends in Caribbean Paleoproductivity related to the Neogene closure of the Central American Seaway. Mar. Micropaleontol. 63: 57-74.

Kameo, K. 2002. Late Pliocene Caribbean surface water dynamics and climatic changes based on calcareous nannofossil records. Palaeogeogr. Palaeocl. 179: 211-226.

Kamikuri, S., I. Motoyama, H. Nishi \& M. Iwai. 2009. Evolution of Eastern Pacific Warm Pool and upwelling 
processes since the middle Miocene based on analysis of radiolarian assemblages: Response to Indonesian and Central American Seaways. Palaeogeogr. Palaeocl. 280: 469-479.

Kriz, S.J. 1990. Tectonic evolution and origin of the Golfo Dulce gols placers in southern Costa Rica. Rev. Geol. Am. Central 11: 27-40.

Kolarsky, R.A., P. Mann \& W. Montero. 1995. Island arc response to shallow subduction of the Cocos Ridge, Costa Rica, p. 235-62. In P. Mann (ed.). Geologic and Tectonic Development of the Caribbean Plate Boundary in Southern Central America. Geol. Soc. Am., Special Paper.

Kwiecinski, B. \& B. Chial. 1983. Algunos aspectos sobre la oceanografía del Golfo de Chiriquí, su comparación con el Golfo de Panamá. Rev. Biol. Trop. 31: 323-325.

Laguna, J. 1985. Systematics, Ecology and Distribution of Barnacles (Cirripedia; Thoracica) of Panama. M.Sc. Thesis, Universidad de California, San Diego, California, EEUU.

Laurito, C. \& A.L. Valerio. 2008. Ictiofauna de la localidad San Gerardo de Limoncito, Formación Curré, Mioceno superior, cantón de Coto Brus, provincia de Puntarenas, Costa Rica. Rev. Geol. Am. Central 39: 65-85.

Lep》, J. \& P. 『milauer. 2003. Multivariate analysis of ecological data using CANOCO. Cambridge University, Cambridge, Reino Unido.

León-Rodríguez, L. 2007. Benthic foraminiferal record of the Pleistocene uplift of the sedimentary deposits of the Burica Peninsula (Costa Rica-Panama) as a result of Cocos Ridge subduction beneath the Central American Arc. MSc. Thesis, Florida International University, Miami, Florida, EEUU.

León-Rodríguez, L. \& L. Collins. 2005. Large paleobathymetric changes indicate rapid pleistocene tectonic uplift of the pacific margin of western Panama-eastern Costa Rica. Geological Society of America Annual Meeting, Abstracts with Programs 37: 160.

Maté, J. 2003. Corals and corals reefs of the Pacific coast of Panama, p. 387-417. In J. Cortés (ed.). Latin American Coral Reefs. Elsevier Science, Amsterdam, Holanda.

Newman, W.A. \& A. Ross. 1976. Revision of the balanomorph barnacles; including a catalogue of the species. Memoirs of the San Diego Society Natural History 9: 1-108.

O’Dea, A. \& J.B.C. Jackson. 2009. Environmental change drove macroevolution in cupuladriid bryozoans. Proc. R. Soc. B. 276: 3629-3634.
O'Dea, A., J.B.C. Jackson, H. Fortunato, J.T Smith, L. D'Croz, K.G. Johnson \& J.A. Todd. 2007a. Environmental change preceded Caribbean extinction by 2 million years. Proc. Natl. Acad. Sci. USA. 104: 5501-5506.

O’Dea, A., F. Rodríguez, C. De Gracia \& A. Coates 2007b. La paleontología marina en el Istmo de Panamá. Canto Rodado 2: 149-179.

Olsson, A.A. 1942. Tertiary and Quaternary fossils from the Burica Peninsula of Panama and Costa Rica. Bull. Am. Paleontol. 27: 157-258.

Porter, J.W. 1972. Ecology and species diversity of coral reefs on opposites sides of the Isthmus of Panama, $\mathrm{p}$. 89-116. In M.L. Jones (ed.). The Panamic biota: some observations prior to sea-level Canal. Bull. Biol. Soc. Wash., Washington D.C., EEUU.

Shipley, T. \& G. Moore. 1986. Sediment accretion, subduction, and dewatering at the base of the slope off Costa Rica: a seismic reflection view of the decollement. J. Geophys. Res. 91: 412-432.

Sibaja-Cordero, J. \& J. Cortés. 2008. Vertical zonation of rocky intertidial organisms in a seasonal upwelling area (Eastern Tropial Pacific), Costa Rica. Rev. Biol. Trop. 56: 91-104.

Smith, T. \& J.B.C. Jackson. 2009. Ecology of extreme faunal turnover of tropical American scallops. Paleobiology 35: 77-93.

Surlyk, F. 1972. Morphological adaptations and population structures of the Danish chalk brachiopods (Maastrichtian, Upper Cretaceous). Biol. Medd. - K. Dan. Vidensk. Selsk. 19: 1-57.

Teranes, J.L., D.H. Geary \& B.E. Bemis. 1996. The oxygen isotope record of seasonality in Neogene bivalves from the Central American Isthmus, p. 105-129. In J.B.C. Jackson, A.F. Budd \& A.G. Coates (eds.). Evolution and Environment in Tropical America. University of Chicago, Chicago, EEUU.

Terry, R.A. 1941. Notes on submarine valleys of Panamanian coast. Geogr. Rev. 31: 337-384.

Todd, J.A., J.B.C. Jackson, K.G. Johnson, H. Fortunato, A. Heitz, M. Alvarez \& P. Jung. 2002. The ecology of extinction: molluscan feeding and faunal turnover in the Caribbean Neogene. Proc. R. Soc. B. 269: 571-577.

Walker, J.D. \& J.W. Geissman (compiladores). 2009. Geologic time scale. Geol. Soc. Am. DOI:10.1130/2009. CTS004R2C. 